\title{
REFLEXIONES DE LOS VOLCANÓLOGOS SOBRE SU PAPEL PROFESIONAL EN LA GESTIÓN DEL RIESGO VOLCÁNICO EN EL CONTEXTO DE AMÉRICA LATINA
}

\author{
REFLECTIONS OF VOLCANOLOGISTS ABOUT THEIR \\ PROFESSIONAL ROLE IN VOLCANIC RISK MANAGEMENT \\ IN THE CONTEXT OF LATIN AMERICA
}

\author{
Dolors Ferrés ${ }^{1 *}$, Rudiger P. Escobar ${ }^{2} \&$ Luke Bowman $^{2}$ \\ ${ }^{1}$ Departamento de Vulcanología. Instituto de Geofísica. Universidad Nacional \\ Autónoma de México \\ ${ }^{2}$ Department of Geological and Mining Engineering and Sciences. Michigan \\ Technological University \\ * Autora para contacto: dferres@geofisica.unam.mx
}

(Recibido: 12/08/2011; aceptado: 29/03/2015)

\begin{abstract}
The strengths and common challenges faced by Latin American volcanologists that assume a variety of roles in the volcanic risk reduction context were discussed in the workshop "PASI 2011: Open Vent Volcanoes ". The event was held in Costa Rica with the participation of professionals from most countries of the American Pacific coast plus Argentina, the UK, Norway and Sweden. Several issues were specifically addressed, which allowed to discuss needs in five topics: 1) Lack of financial, human and technical resources at the observatories in the Latin American region; 2) Governmental and non-governmental programs' cooperation between technical and academic organizations; 3) Need for regional coordination, 4) The role of volcanologists as communicators and their interaction with other stakeholders in volcanic risk management, and 5) The contribution of the social sciences and the creation of multidisciplinary groups in the volcanological observatories.

The Pacific margin of the American continent is an area highly exposed to volcanic activity, including some of the most important cities in the continent. Nevertheless, the institutions in charge of studying and monitoring the volcanic activity and hazards in many of the countries of this region face severe limitations to carry out their work. For the majority of Latin American countries, the large number of potentially active volcanoes and the reduced number of volcanologists who work in their monitoring, become one of the main limitations. The cooperation between observatories, and with universities and research centers, has helped to cover this need, at least partially. Beyond their scientific and technical role as physical and natural sciences experts, the volcanologists have also been involved in tasks that require the development of other skills, mainly as communicators of information related to volcanic risk. This has led to an increase in
\end{abstract}

FERRÉS, D., ESCOBAR, R. P. \& BOWMAN, L., 2015: Reflexiones de los volcanólogos sobre su papel profesional en la gestión del riesgo volcánico en el contexto de América Latina.- Rev. Geol. Amér. Central, 52: 9-31, DOI: 10.15517/rgac.v0i52.18227 
multidisciplinary collaboration with scientists from the social field. However, there are still large challenges that need to be addressed in the near future. The contributions of the discussion sustained at the workshop can act as a starting point for future forums and actions aimed at reducing risk associated with volcanic activity.

Key words: volcano observatories, Latin America, roles, risk management, strengths and weaknesses.

RESUMEN: Las fortalezas y problemáticas comunes de los volcanólogos en los diversos roles que desempeñan como actores para la reducción del riesgo volcánico en América Latina fue debatida en el marco del taller "PASI 2011: Volcanes de conducto abierto", celebrado en Costa Rica, con participación de profesionales de casi todos los países de la por "costa pacífica del continente, además de Argentina, el Reino Unido, Noruega y Suecia. Se trataron gran diversidad de temas, agrupados en cinco bloques, que permitieron definir necesidades comunes en cinco ámbitos : 1) Carencia de recursos financieros, humanos y técnicos en los observatorios de la región; 2) Programas de cooperación gubernamental y no gubernamental entre organismos técnicos y académicos; 3) Necesidades de coordinación regional; 4) El rol de los volcanólogos como comunicadores y su interacción con otros actores de la gestión del riesgo y 5) El aporte de las ciencias sociales y la creación de grupos multidisciplinarios en el ámbito de los observatorios volcanológicos.

La margen pacífica del continente americano es un área altamente expuesta a la actividad volcánica, incluyendo algunas de las ciudades más importantes en el continente. Sin embargo, las instituciones encargadas de la observación y el monitoreo de la actividad y las amenazas volcánicas en muchos de los países de esta región, enfrentan serias limitaciones para desempeñar su labor. Para la mayoría de países latinoamericanos, el gran número de volcanes potencialmente activos y el reducido número de volcanólogos que trabajan en su monitoreo, constituyen una de las principales limitantes. La cooperación entre observatorios con universidades y centros de investigación, ha ayudado a cubrir esta necesidad al menos de forma parcial. Trascendiendo su papel científico y técnico como expertos en ciencias físicas o naturales, los volcanólogos también se han visto involucrados en tareas que requieren el desarrollo de otras habilidades, principalmente como comunicadores de información sobre el riesgo volcánico. Esto ha llevado a incrementar la colaboración multidisciplinaria con profesionales del área de las ciencias sociales. Sin embargo, existen aún grandes retos que deberán abordarse en un futuro próximo. Los aportes de la discusión sostenida en el taller pueden constituir un punto de partida para futuros foros y acciones orientados a la reducción del riesgo asociado con la actividad volcánica.

Palabras clave: Observatorios volcanológicos, América Latina, roles, gestión de riesgos, fortalezas y debilidades.

\section{INTRODUCCIÓN}

En enero del 2011, 60 representantes de diversas instituciones académicas del ámbito de la geología y de observatorios volcanológicos de 16 países celebraron en la Universidad de Costa Rica (San José) un taller para analizar los avances en la vigilancia de volcanes activos en América Latina y conocer técnicas de monitoreo volcánico novedosas que usan los sensores remotos para la obtención de información en los volcanes. El taller "PASI 2011: Volcanes de Conducto Abierto" fue organizado por la Fundación Nacional de Ciencias de los Estados Unidos por medio de la Universidad Tecnológica de Michigan, en colaboración con la Escuela Centroamericana de Geología de la Universidad de Costa Rica. Se reunieron expertos de un amplio rango de disciplinas para discutir temas relacionados con los esfuerzos que cada país está realizando en la me- jora de la vigilancia volcánica y la evaluación de amenazas, a través de la implementación de técnicas de monitoreo de vanguardia, y ofrecer una mejor información sobre el estado de los volcanes activos. Todos los países de la costa pacífica del continente y Argentina estuvieron representados por participantes pertenecientes a observatorios, universidades e instituciones de investigaciones en cada uno de estos países (Fig. 1). También participaron científicos del Reino Unido, Noruega y Suecia.

La cuarta jornada del taller fue dedicada al análisis del aporte de la volcanología a la gestión del riesgo y al debate sobre los diferentes roles de los volcanólogos en tiempos de tranquilidad y de crisis volcánica, así como las dificultades para desarrollarlos (Rose \& Palma, 2011). Este artículo ofrece un resumen de la valiosa discusión mantenida, en la cual se abordaron diversos temas de importancia relacionados con las labores 


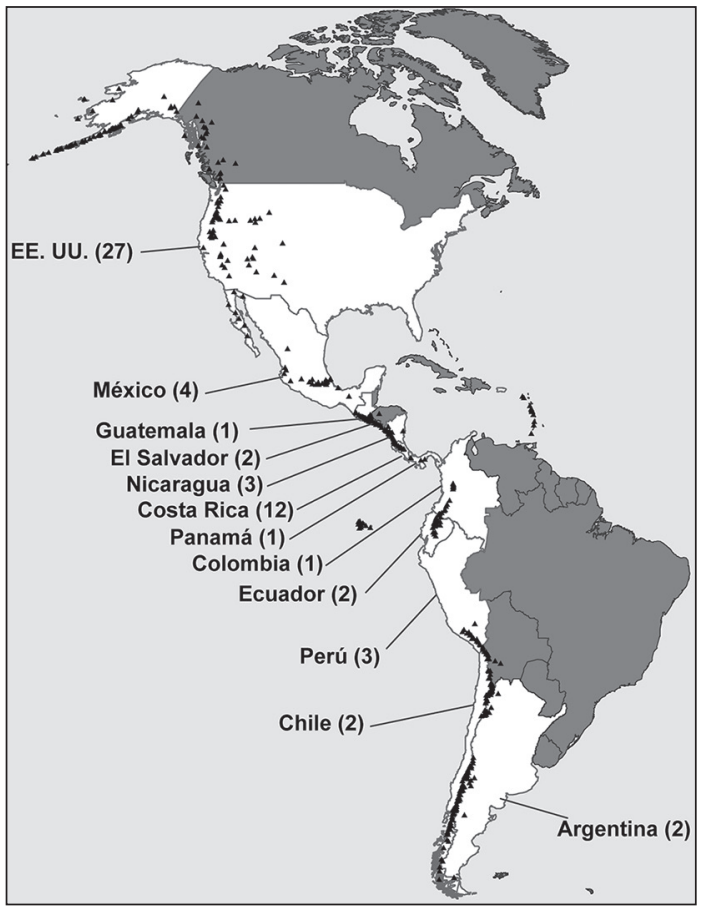

Fig. 1: Mapa de los países del continente americano representados en el taller "PASI 2011: Volcanes de Conducto Abierto". Junto al nombre del país y entre paréntesis, se indica el número de participantes. Los triángulos negros representan las ubicaciones de los volcanes activos durante el Holoceno, según la base de datos del Global Volcanism Program (Venzke et al., 2009).

técnicas y educativas que los volcanólogos desempeñan en los observatorios de la región, así como su relación con instituciones académicas y de cooperación.

Presentamos los puntos claves y comunes que se identificaron durante el panel de discusión mantenido en la última parte de la jornada, después de las presentaciones ofrecidas por los volcanólogos de diferentes países de América Latina (Nicaragua, Colombia, Ecuador, El Salvador). El panel permitió la participación de todos los presentes, que aportaron su opinión sobre la realidad de los observatorios en la región y sobre otros cinco aspectos principales:

1. Las áreas volcánicas como riesgo y como recurso: oportunidades para la población y la exposición a las amenazas volcánicas.

2. Roles de los volcanólogos en el ámbito técnico y educativo.
3. Cooperación institucional gubernamental y no gubernamental (ONG) para la reducción del riesgo asociado con la amenaza volcánica.

4. Redes de comunicación. Generación de información sobre las amenazas y su traslado a los posibles usuarios.

5. Las implicaciones humanas y sociales de las amenazas volcánicas, desde el punto de vista de la gestión y la percepción del riesgo.

Los volcanólogos de la región latinoamericana han realizado esfuerzos en las tres últimas décadas para desarrollar algunos de los problemas y necesidades en la vigilancia volcánica, el análisis de la peligrosidad volcánica en cada país y en general de los diferentes roles que deben abordar en su labor en los observatorios. Newhall et al. (1999) ya abordaron la problemática de los diferentes roles de los profesionales de la volcanología en el manejo de crisis volcánicas. Las ideas expresadas en el taller constituyen un aporte de gran interés a este debate y lo amplía, pudiendo así constituir el punto de partida para la creación de un marco de referencia común que establezca el "dónde estamos" y "hacia dónde queremos ir" en el ámbito de la reducción de riesgo asociado con la actividad volcánica.

Aunque los expertos participantes expresaron diferentes puntos de vista en diversos de los temas tratados, se identificaron también muchos puntos en común. Centrarse en estas coincidencias y analizarlas en profundidad podría hacer posible el crear nuevas formas de colaboración entre los observatorios de la región y entre estos y las instituciones académicas (universidades latinoamericanas y extranjeras). Esto también podría ayudar a definir las formas de relación con los organismos de cooperación gubernamental y no gubernamental, con el fin de ir avanzando en un proceso de construcción de relaciones más sostenibles, que aunque lento y dilatado en el tiempo, permita la mejora del trabajo de los volcanólogos en la región y se traduzca en acciones concretas de reducción de riesgo de origen volcánico.

El artículo no presenta datos recolectados de forma sistemática, ni un análisis científico riguroso que pretenda probar alguna hipótesis específica, pero aporta una descripción de la discusión y el análisis informal que los participantes del taller 
sostuvieron, así como las conclusiones preliminares a las cuales los autores del artículo arribaron. Este texto sigue una narrativa más bien informal y no pretende ser exhaustivo, pero tiene como finalidad describir los principales ejes del debate mantenido, que pueden constituir el punto de partida para un tratamiento más amplio en futuros foros (presenciales o virtuales) de volcanólogos en la región, que integren, de ser posible, a otros colegas que no pudieron participar en este taller y animen a incorporar otras personas interesadas en la coordinación de esfuerzos, como por ejemplo, representantes de Protección Civil y autoridades locales de áreas expuestas a la amenaza volcánica, entre otros.

\section{COINCIDENCIAS DE LA REALIDAD DE LOS OBSERVATORIOS VOLCANOLÓGICOS DE LA REGIÓN LATINOAMERICANA}

Las charlas ofrecidas durante la jornada de discusión evidenciaron que los países de América Latina tienen capacidades humanas e instrumentales diferentes para el desarrollo de las labores de monitoreo y evaluación de las amenazas volcánicas, pero tienen también fortalezas y limitantes comunes.

Las diferentes capacidades de los observatorios son función principalmente del tiempo que los observatorios llevan funcionado y de los recursos que tienen asignados. Estos recursos no siempre son proporcionales al número de volcanes que es necesario vigilar, ni a los niveles de actividad volcánica en cada país. Así por ejemplo, Guatemala con dos o tres volcanes en erupción casi de forma permanente, tiene solamente dos volcanólogos de planta en las oficinas centrales de la organización encargada de la observación volcanológica (Instituto Nacional de Sismología, Vulcanología, Meteorología e Hidrología: INSIVUMEH), y uno o dos volcanólogos en cada uno de los observatorios situados en las comunidades cercanas a los volcanes. En general, los países de Sudamérica o México tienen más capacidades instrumentales en relación con América Central (es el caso de Ecuador, p.ej.). La Asociación Latinoamericana de Volcanología (ALVO), creada en el 2010, ha emprendido un análisis en profundidad de las capacidades, fortalezas, oportunidades y debilidades en las instituciones responsables de la volcanología en los diferentes países de la región (ver artículo de Delgado-Granados et al., 2015).

Muchos observatorios volcanológicos tienen en común la imposibilidad de tener personal especializado en una sola técnica de monitoreo o personal desarrollando investigación. Actualmente, estas y otras limitantes representan una fuerte debilidad en el desempeño del monitoreo volcánico en América Latina, comparado con el desarrollo de la vigilancia volcánica en otras zonas (p. ej.: Europa y EE. UU.). En el taller se puso de manifiesto cómo esta situación hace necesario que los técnicos y especialistas trabajando en volcanología asuman diferentes roles, especialmente en periodos de crisis eruptivas, tanto en el ámbito técnico de obtención y análisis de datos volcanológicos, como en el ámbito educativo, con la preparación de escenarios eruptivos y su divulgación. Se señaló con amplio acuerdo, la necesidad de identificar las lagunas de conocimiento en cada observatorio y establecer relaciones de trabajo con las instituciones académicas que permitan complementar las carencias en cada institución. En general, los observatorios vinculados a universidades tienen acceso a más recursos para la investigación y por lo tanto, pueden desarrollar mayores capacidades para desempeñar sus funciones. Los observatorios de la mayor parte de los países de América Latina han establecido relaciones con grupos de trabajo extranjeros para investigaciones puntuales y el desarrollo de sistemas y redes de instrumentación, que como el caso de Colombia, han sido claves para la conformación, fortalecimiento y capacitación del grupo nacional de volcanólogos. En algunos países, los observatorios han establecido acuerdos de colaboración con las universidades nacionales, pero en muchas ocasiones a un nivel de trabajo personal, no institucionalizado, lo cual dificulta la continuidad de las acciones conjuntas. 
Por otro lado, una fortaleza que es común al personal de muchos de los observatorios volcanológicos de la región, es la amplia gama de formaciones y experiencia profesionales, provenientes de una diversidad de campos técnicos y científicos, que incluyen a geólogos, químicos, físicos, etc., así como a personal técnico formado en áreas de ciencia aplicada e ingeniería (p. ej.: ingenieros civiles, electrónicos, mecánicos, de computación). Uno de los retos planteados es cómo aprovechar y complementar este amplio rango de experiencias en cada institución a nivel nacional y regional para poder lograr objetivos similares de una manera más eficiente.

Otro aspecto común es que cada país latinoamericano tiene experiencias muy valiosas en sus historias de crisis volcánicas. Cada una de ellas podría compartirse con otros países, por lo que se puso de relieve la necesidad de sistematizar estas experiencias y analizar las "lecciones aprendidas" en cada una de ellas. Las erupciones recientes son una oportunidad para evaluar el funcionamiento de protocolos de actuación y de comunicación, los cuales deberán adaptarse de forma óptima al comportamiento y la percepción de riesgo por parte de la población. Este último aspecto, cuyo estudio obliga necesariamente a los volcanólogos a colaborar con especialistas del área de las ciencias sociales, es el menos explorado por el momento y plantea importantes retos para una transmisión efectiva de la información volcanológica que se genera para la población. Por ejemplo, en el caso de Guatemala, donde actualmente tres volcanes se encuentran en erupción con actividad de baja intensidad, pero que ocasionalmente se incrementa hasta el punto de causar crisis de riesgo, presenta una buena oportunidad para estudiar el tema del comportamiento y la percepción del riesgo, en escenarios reales de riesgo con crisis volcánicas. Aprovechar este tipo de "laboratorios naturales" para desarrollar el conocimiento que permita mejorar el actuar de los distintos entes y personas que participan en la gestión del riesgo volcánico, podría ser de gran valor práctico.

\section{LAS ÁREAS VOLCÁNICAS COMO RIESGO Y COMO RECURSO: OPORTUNIDADES PARA LA POBLACIÓN Y EXPOSICIÓN A LAS AMENAZAS VOLCÁNICAS}

La mayor parte de los países latinoamericanos, especialmente en América Central, exhiben un patrón similar de alta exposición a la amenaza volcánica, puesto que sus principales ciudades y centros de población están ubicados próximos a las cordilleras volcánicas. La formación del paisaje y del relieve por procesos volcánicos ha favorecido el asentamiento de una gran parte de la población en áreas volcánicas. Por ejemplo la formación de valles de fondo plano rellenados por voluminosas ignimbritas (que en muchos casos resultan ser excelentes acuíferos) crea un espacio geográfico muy atractivo para el asentamiento de grandes centros urbanos. De forma similar, algunos de los mejores suelos agrícolas, muchas veces ubicados en zonas con excelente acceso a recursos hídricos, y con condiciones climatológicas favorables determinadas por el relieve volcánico (p. ej.: la elevación y por lo tanto la precipitación y temperatura) se encuentran en áreas de volcanismo reciente o activo. Esto ha favorecido que las comunidades se asienten en las llanuras entre volcanes y se extiendan hasta las laderas de los edificios volcánicos, y en la proximidad o el interior de algunas calderas, que aún podrían considerarse como sistemas volcánicos activos. Además, los volcanes representan, en muchos casos, una fuente de ingresos, pudiendo ser explotados como áreas turísticas o de producción de energía geotérmica.

Varias de las presentaciones ofrecidas durante el taller abordaron el tema de la exposición de la población a la amenaza volcánica, con intervenciones de los participantes que presentaron casos específicos de crisis volcánicas y condiciones de riesgo asociadas con algunos de los volcanes más activos en América Latina. Estas permitieron la introducción y fueron el punto de partida para la discusión sobre las amenazas volcánicas y su 
relevancia para la sociedad, la mitigación de riesgos y el papel que los volcanólogos desempeñan en la reducción y gestión del riesgo.

\section{ROLES DE LOS VOLCANÓLOGOS DE LA REGIÓN LATINOAMERICANA}

La finalidad del trabajo de los volcanólogos que trabajan en gestión del riesgo, debe estar enfocada a minimizar los efectos de la actividad volcánica sobre las personas y sus bienes y en última instancia, a salvar vidas. Para la mayor parte de los profesionales de la volcanología, es claro que este fin común no puede ser abordado exclusivamente por los observatorios, sino que es necesario el engranaje de una red de actores y de instituciones (técnicas, académicas, de protección civil, ciudadanas y de gobierno) entre los cuales exista una comunicación clara, fluida y organizada.

Algunos participantes apuntaron que la realidad de cada país y de cada profesional en concreto es diferente en función de su ubicación, de la situación de la actividad volcánica en un tiempo determinado y de su formación. Esto hace imposible generalizar el rol y funciones de los volcanólogos, cada uno con sus propias fortalezas y debilidades y con requerimientos institucionales y necesidades diferentes. En un plano ideal, cada profesional debería poder desarrollar aquellas funciones o actividades que mejor se adapten a su formación, interés, personalidad y capacidades. Sin embargo, como quedó de manifiesto en las diversas presentaciones de la jornada de debate, la carencia de recursos humanos y de medios en muchos países de la región hace necesario que los profesionales se enfrenten a una gran diversidad de funciones y de roles, para algunos de los cuales no han recibido formación específica, y en los que muchas veces no se sienten preparados.

En este marco, las diferentes actividades que se desarrollan en los observatorios volcanológicos de la región pueden englobarse en tres funciones principales:

a) Vigilar los volcanes activos en cada país: adquirir, analizar e interpretar datos que se obtienen de las diferentes técnicas de monitoreo volcánico, para la caracterización de líneas base de actividad volcánica para cada uno de los principales volcanes activos. Son diversas las técnicas que se desarrollan, con diferentes grados de aplicación en cada volcán y en cada país. Las mejor implementadas son la vigilancia sísmica, la vigilancia de gases y en menor grado el monitoreo de deformación, el monitoreo hidrogeoquímico y de temperaturas (gases y aguas). La instrumentación necesaria para desarrollar una determinada técnica de monitoreo es adquirida por los diferentes países de dos formas principales: con recursos propios de las instituciones gubernamentales o académicas donde están adscritos los observatorios, a petición de los volcanólogos a través de los planes anuales de trabajo, o más comúnmente a través de proyectos específicos o a través de cooperación internacional de tipo gubernamental. La relación de los grupos de vigilancia volcánica de la región con grupos de universidades extranjeras ha posibilitado la capacitación de diversos técnicos en los observatorios y en algunos casos la especialización en instituciones académicas de postgrado. La aplicación de técnicas de monitoreo específicas ha sido posible gracias a estos tipos de formación, que incluye la instalación y uso de la instrumentación, la obtención y el análisis de datos, y su interpretación. Sin embargo, en muchos casos esta capacitación no es sistematizada documentalmente, de forma que el aprendizaje queda en las personas y no en los grupos de trabajo e instituciones.

b) Evaluar la peligrosidad de los principales volcanes activos: realizar estudios de estratigrafía volcánica, caracterización de la actividad eruptiva pasada y modelación de procesos volcánicos para la construcción de mapas de amenaza y escenarios de riesgo. En las últimas dos décadas se han tenido avances en esta área, principalmente a través de la colaboración de los observatorios con centros de investigación extranjeros (p. ej.: de países centroamericanos con México, Norte América (EE. UU. y Canadá), etc.). Generalmente el recurso humano de las áreas de volcanología de los observatorios es insuficiente para abordar este trabajo en solitario.

c) Divulgar el estado de conocimiento sobre los principales volcanes activos: elaborar árboles de probabilidad de escenarios de actividad 
volcánica y establecer protocolos de actuación y comunicación en caso de crisis volcánica. En épocas de tranquilidad volcánica la divulgación se realiza principalmente a través de las ciberpáginas de los observatorios, así como a través de charlas o folletos. Sin embargo, en caso de crisis volcánica, la divulgación de la actividad volcánica involucra a otros tres actores principales (pero no los únicos): personal de comunicación o de gestión de riesgos de los propios observatorios, las autoridades de defensa o protección civil y los medios de comunicación. El papel del volcanólogo como comunicador, los productos divulgativos que se elaboran en los observatorios, los protocolos de comunicación entre los actores mencionados y el reto de hacer llegar la información sobre volcanología y amenaza volcánica a las comunidades y sus organizaciones, fueron temas ampliamente debatidos durante la jornada. Algunos de los aspectos puestos de relieve se desarrollan en los apartados siguientes.

Además de estas responsabilidades y de las actividades asociadas con ellas, quedó plasmado en la discusión que los diferentes observatorios realizan otros esfuerzos a los cuales es necesario destinar una buena parte de tiempo, incluyendo:

d) Relación directa con la población: diversos asistentes propusieron que el papel del volcanólogo incorpora o debe incorporar estrategias para ganar la confianza de la población en las comunidades donde se trabaja y que podrían resultar afectadas por la actividad volcánica. Esto requiere, por una parte, desarrollar capacidades de comunicación, teniendo en cuenta aspectos culturales y de cosmovisión de los grupos humanos a los que los profesionales se dirigen y por otra parte la capacidad de recibir y asimilar la información que proviene de ellos, para hacer bidireccional la relación entre los expertos y la comunidad. Se planteó también la problemática de que esta comunicación directa pueda desbordar las capacidades de los observatorios y estar asumiendo responsabilidades y funciones de otras instituciones, como los organismos de protección civil. Como ejemplo, un caso de El Salvador durante la crisis del Volcán de Santa Ana en 2005. Los volcanólogos visitaron frecuentemente algunas comunidades al pie de la ladera sur del volcán (p. ej.: San
Blas, San Isidro) para realizar las actividades de monitoreo. Para muchas personas ese contacto les proveía de la única información proporcionada por un ente oficial, y esto ayudó a crear un clima de confianza de la población respecto a la institución técnica. Sin embargo, a medida que la crisis volcánica avanzaba, las demandas de información abarcaban aspectos del ámbito de la logística y de la protección civil que los volcanólogos no podían responder.

e) Relación directa con gobiernos locales y organismos de cooperación: se discutió también la necesidad de que los volcanólogos se involucren en coordinar y colaborar con otros organismos y con los gobiernos locales que están presentes en las áreas de influencia de los volcanes, para optimizar el trabajo y evitar duplicación de esfuerzos, así como para apoyar a las autoridades en la transmisión de la información a la población sobre peligro volcánico y riesgo en general. Las colaboraciones entre organismos, aunque costosas en tiempo y esfuerzos, permiten amplificar de una forma más eficiente el trabajo de cada institución.

Generalmente, el recurso humano que integra las áreas de volcanología de los diferentes observatorios, es insuficiente para desarrollar de forma óptima el conjunto de estas funciones. Por otra parte, la remuneración económica que percibe la mayor parte de este personal es poco o nada competitiva. Cabe señalar finalmente, que los observatorios cuentan con poco personal dedicado a tareas de mantenimiento de los equipos, por lo que frecuentemente el personal de las áreas de volcanología debe involucrarse en estas tareas.

\section{COOPERACIÓN INSTITUCIONAL GUBERNAMENTAL Y NO GUBERNAMENTAL (ONG) PARA LA REDUCCIÓN DEL RIESGO ASOCIADO CON LA AMENAZA VOLCÁNICA}

Una buena parte del avance en las capacidades instrumentales, técnicas y de capacitación del personal de los observatorios en la región en los últimos años ha sido posible gracias a proyectos de cooperación internacional y a colaboraciones 
entre las instituciones. Esta coincidencia de los expertos participantes se manifestó en la discusión mantenida. Sin embargo, tal y como se ejemplifica más adelante, se evidenciaron también algunas de las problemáticas que determinados tipos de proyectos, o de cooperación en general, pueden suponer en el funcionamiento cotidiano de los observatorios.

Los observatorios han desarrollado una red de colaboraciones, en parte voluntarias, para cubrir parte de sus necesidades. Sin embargo, una de las principales problemáticas relacionadas es la no institucionalización de estas relaciones de trabajo, que dependen de un experto en específico y no están formalizadas a nivel de las instituciones. En el nivel nacional pueden identificarse cuatro tipos de cooperaciones o colaboraciones principales: a) la relación con instituciones nacionales afines al campo de la volcanología, b) la colaboración con universidades nacionales y extranjeras, c) la relación con organismos de cooperación no gubernamental, y d) la colaboración con las comunidades en áreas expuestas a la amenaza.

La mayor parte de observatorios de la región ha establecido vínculos de trabajo con las universidades nacionales, especialmente en aquellos casos, donde estas últimas desarrollan estudios de geología, geofísica y gestión de riesgos, y en algunos casos con la industria eléctrica (Costa Rica) o geotérmica (Nicaragua, El Salvador), donde existe un nutrido grupo de profesionales trabajando en estas áreas. En algunos casos, como el Servicio Nacional de Sismología y Vulcanología del Instituto Geofísico de la Escuela Politécnica Nacional (IG EPN) en Ecuador o el Observatorio Vulcanológico y Sismológico de Costa Rica (OVSICORI), están directamente vinculados con las universidades nacionales. Aquellos observatorios que no están directamente asociados a organismos académicos tienen grandes limitantes y muy pocas posibilidades de desarrollar investigación pura y trabajos en profundidad de caracterización de la actividad eruptiva y de evaluación de la amenaza volcánica. Como ya se citó, estas carencias han sido cubiertas en gran parte con proyectos de cooperación con universidades extranjeras.
La colaboración ha sido principalmente con diversos grupos de investigación académica estadounidenses, europeos y en menor medida japoneses. Sin embargo, en la mayor parte de los casos, estas colaboraciones son puntuales y requieren que los volcanólogos de los países latinoamericanos destinen mucho tiempo a la asistencia de los colegas extranjeros. En muchas ocasiones los resultados de este trabajo en común (capacitación, productos científicos en forma de artículos principalmente) no son incorporados al quehacer del observatorio, ni significan mejoras significativas en el conocimiento que resulta más urgente recabar (p. ej.: aspectos de amenaza) de los volcanes de los que se está a cargo. En los últimos años, los grupos de investigación extranjeros han practicado una cultura de colaboración y de asistencia técnica que permite un mayor acercamiento entre los investigadores y un mayor involucramiento del personal de los observatorios de la región. Sin embargo, todavía es difícil congeniar los diferentes fines de los observatorios (técnico-operativos) y de los grupos de investigación (académicoscientíficos). El idioma ha sido tradicionalmente un problema para el desarrollo de los trabajos en común, pero cada vez más, los investigadores extranjeros hablan español y los volcanólogos en América Latina manejan el inglés.

Por otra parte, en los diferentes países de la región existe la presencia de numerosas organizaciones no gubernamentales que desarrollan sus proyectos de cooperación en multitud de ámbitos, algunos de los cuales tienen líneas de trabajo en común con las funciones de los volcanólogos en los observatorios. Se trata principalmente de aquellas organizaciones y proyectos que desarrollan acciones para la organización y capacitación de las comunidades rurales en gestión de riesgos y en la implementación de sistemas de alerta temprana. Los participantes manifestaron que muchas de estas acciones no han contado en su planificación y ejecución con las líneas de trabajo ya establecidas y los productos de evaluación de amenazas elaborados en las instituciones nacionales responsables de la gestión de riesgos, provocando duplicación de esfuerzos y en algunos casos "contradicción" o conflicto en los productos resultantes. Algunos observatorios, como es el caso de INETER en 
Nicaragua, han destinado parte de sus esfuerzos en corregir estas situaciones, tratando de acercar los objetivos de los proyectos de cooperación de los organismos no gubernamentales a los objetivos del observatorio, sumando de esta forma los esfuerzos de ambos.

En los últimos años se han percibido mejoras tanto en la capacidad de las instituciones nacionales (observatorios) para demandar sus necesidades, como en el grado de responsabilidad de los organismos no gubernamentales en adecuar sus programas y proyectos a la realidad del territorio donde van a implementarlos. Sin embargo, los lineamientos y requerimientos de los programas de cooperación (tiempos, líneas de actuación), frecuentemente diseñados en los países de origen de las organizaciones no gubernamentales, impiden a las contrapartes locales una mejor adaptación de los proyectos a las necesidades reales de la población beneficiaria y establecer coordinaciones a nivel interinstitucional (Bowman \& White, 2012).

Finalmente, diferentes observatorios de la región han desarrollado "programas de observadores locales" para lograr tener presencia en las áreas de influencia de diferentes volcanes activos dentro de su territorio y poder recabar información insitu, con el objetivo de cubrir ciertas carencias que se presentaban al estar las oficinas de los observatorios centralizadas en las capitales de los países. Es el caso, entre otros, de Ecuador, Guatemala y El Salvador. Otros países como Colombia y Costa Rica han fortalecido sus observatorios con diferentes sedes en diferentes puntos del país. Los aspectos de comunicación relacionados con estas estructuras descentralizadas se discute en los siguientes capítulos.

En el nivel regional se identifican igualmente dos tipos de colaboraciones: a) la relación entre especialistas en la región y b) la implementación de proyectos a nivel regional.

Los técnicos de las áreas de volcanología han mantenido relación con otros de diferente nacionalidad a nivel personal para discutir aspectos específicos de sus tareas. En ocasiones, como en el caso de crisis volcánicas, esta relación personal se ha intensificado, integrando puntualmente a un colega en el comité técnico de crisis vía correo electrónico. En otras ocasiones, se han establecido colaboraciones puntuales para realizar trabajo de campo (apoyo de Guatemala a El Salvador para realizar mediciones de gases por medio de COSPEC, o de El Salvador a Nicaragua para realizar mediciones con cámara térmica, p.ej.). Sin embargo, no existe actualmente un organismo que constituya un comité técnico regional en caso de crisis volcánicas, siendo necesario apoyarse en los comités internacionales como los del Volcanic Disaster Assistance Program (VDAP). La ALVO, tiene entre sus principales objetivos solventar esta situación, pero se plantea el problema de los recursos que puedan destinarse a este fin. Entre las acciones que un comité de este tipo debería abordar están: i) aprendizaje en el uso de nuevas técnicas de monitoreo; ii) compartir datos, información y análisis de la vigilancia de un volcán en específico; iii) establecer pronósticos de actividad volcánica, con base en criterios comunes del peso relativo de la información proporcionada por cada técnica de monitoreo, y iv) vigilancia de edificios volcánicos cerca o en zonas de fronteras geográficas (p. ej.: Volcán Tacaná en la frontera entre México y Guatemala).

Otro aspecto a impulsar podría ser la creación de un centro-foro virtual de información volcánica en tiempo real donde se pueda estar monitoreando la actividad de cada volcán de la región desde cualquier lugar. Esto requiere que todos los observatorios tengan la tecnología para poner en línea los sismogramas y en general todos los datos en tiempo real de los diferentes tipos de monitoreo que realizan. La autoría o propiedad de los datos, su presentación y accesibilidad para los diferentes tipos de usuarios (científicos, técnicos, comités de protección civil y público en general) y el uso que se realice de la información son temas que será necesario debatir y que algunos observatorios ya han abordado parcialmente en el ámbito nacional, especialmente cuando se trabaja en colaboración con investigadores de instituciones académicas (p.ej: la implementación de algunas de las técnicas de monitoreo que proporcionan datos en tiempo real en el Centro Nacional de Prevención de Desastres (CENAPRED) en México han sido facilitados por investigadores de la UNAM). La red VHUB (http://vhub.org/), 
por ejemplo, cuenta con un proyecto piloto para compartir diversos tipos de información que incluye a observatorios de Sudamérica, el Caribe y Europa. El foro en línea es de especial relevancia para poder preguntar o consultar a colegas de la región cuando un profesional está sumergido atendiendo una emergencia.

La carencia de recurso humano suficiente y su consecuencia directa, la carencia de tiempo, dificultan que los observatorios de la región puedan asimilar de forma práctica los avances en el uso de nuevas técnicas de monitoreo o destinar tiempo a aspectos de coordinación regional. En este punto, un problema que cualquier organización o institución enfrenta es identificar y seleccionar aquellos métodos y técnicas que deberá implementar e incluir en su desempeño, y de las cuales pueda obtener el mayor beneficio, en función de su misión o mandato. Los criterios para definir esto pueden ser muy amplios, pero en el caso de técnicas novedosas o experimentales que son promovidas por investigadores y académicos, es difícil establecer criterios para determinar si estas se adaptan a las necesidades y la realidad de operación de los observatorios en cada país, y si es una buena idea invertir en esas técnicas o tecnología, de tal forma que puedan ser las más útiles para la vigilancia volcánica, en cada caso especifico. Existen dificultades en discernir qué beneficios concretos aporta una nueva técnica de monitoreo, cuál es el valor que ofrece la información que se obtiene de ella y qué peso puede tener en el análisis global de toda la información que proporcionan diferentes técnicas de monitoreo (especialmente las clásicas, como la sísmica). En este sentido, algunos investigadores que trabajan en universidades en países desarrollados, que tienen una perspectiva más académica y que están interesados en realizar una investigación más "pura" (menos interesados en la parte aplicada), quizás no verán el problema desde la misma perspectiva de los científicos y técnicos que trabajan en un observatorio, y para los cuales los aspectos de aplicación y uso práctico del método o la técnica son los criterios más relevantes para juzgar su utilidad o valor. Por ello, es frecuente observar en el funcionamiento de los observatorios, que la implementación de proyectos o programas de cooperación técnica, tiene aplicación y continuidad mientras existe apoyo de recursos externos, pero que se diluye o se pierde cuando estos programas terminan y se retira la cooperación. El desarrollo de tecnología apropiada para estos casos es imperativo, si la investigación realizada en países desarrollados pretende contribuir a los fines prácticos del monitoreo volcánico a corto y mediano plazo.

\section{REDES DE COMUNICACIÓN}

\section{La generación de información sobre la amena- za y su comunicación a los posibles usuarios}

El rol de los volcanólogos como actores principales en el manejo de crisis volcánicas es uno de los aspectos que más se debatió durante la jornada. Como se mencionó, entre las diversas funciones de los volcanólogos están las acciones de divulgación, que necesariamente se intensifican en periodos de intranquilidad o crisis volcánica.

Todos los observatorios de la región han desarrollado, en menor o mayor medida protocolos de actuación interna en caso de crisis volcánica, que indican las acciones a realizar en las diferentes etapas de evolución del fenómeno volcánico (desde la línea base de actividad normal de un volcán hasta el momento de su erupción). Solamente algunos países, como México, Ecuador o Colombia, disponen de protocolos de actuación diferenciados para cada volcán o zonas volcánicas en su territorio. El Salvador tiene un protocolo común para todos los volcanes, que se modifica y adapta en función de los grados de actividad de cada volcán. El grado de desarrollo de los protocolos está condicionado por la existencia o carencia de antecedentes de actividad histórica y del conocimiento de cada volcán.

Todos los protocolos de actuación interna de los observatorios incorporan un conjunto de acciones de comunicación y relación con otras instituciones y actores claves para el manejo de crisis (instituciones científico-técnicas, instituciones de protección civil, medios de comunicación), que buscan una transmisión de la información clara, fluida y organizada. Solamente en algunos casos 
estas acciones se han diseñado de forma conjunta entre las diferentes instituciones involucradas.

Tradicionalmente, el modelo de generación de información sobre la amenaza volcánica y su comunicación a usuarios finales ha sido planteada, con algunas variantes, de forma tal que el observatorio comunica a la institución de protección civil los cambios en los grados de actividad de un volcán, describiendo idealmente de forma explícita (aunque en la práctica se da más frecuentemente de forma implícita) algunas de las posibles consecuencias de la amenaza, y es la institución de protección civil la que traslada la información sobre la actividad del volcán junto con un conjunto de recomendaciones sobre las acciones a realizar, a la población expuesta.

Aceptando este paradigma como el modelo de generación y comunicación, la relación de los observatorios con los organismos de protección civil se basa en los grados de confianza y credibilidad entre las distintas instituciones. Desde muchos observatorios se ha realizado un trabajo de coordinación con la protección civil para crear lazos de confianza, y en muchos casos superar las reticencias o simplemente la falta de interés hacia las instituciones científico-técnicas por parte de instituciones gubernamentales y gobiernos locales, en el tema de la asesoría sobre las amenazas y las crisis volcánicas.

Generalmente, los organismos de protección civil están estructurados en diferentes niveles, desde el ámbito nacional a lo local, con diferentes niveles intermedios según los países. Es frecuente que durante la evolución y ocurrencia de crisis volcánicas, se presenten demandas no coordinadas de información por parte de estos diferentes niveles, con grados de conocimiento y comprensión del fenómeno distintos. Por otra parte, ocurre en muchos países, que existe una formación insuficiente del personal de protección civil en materia de volcanología para comprender los pronósticos de actividad volcánica y los protocolos de actuación interna. Esto se debe, entre otras causas, a que el fenómeno volcánico y sus amenazas no se presentan con la misma frecuencia que otras amenazas, como por ejemplo las hidrometeorológicas (lluvias intensas, inundaciones, etc.), o las geológicas como los flujos de lodo y los deslizamientos, que suelen tener una recurrencia anual durante las estaciones lluviosas en los países latinoamericanos. Por tanto, la capacitación que reciben las instancias de protección civil sobre el origen y evolución del fenómeno volcánico es menor que para el resto de los fenómenos naturales mencionados. Así, en muchas ocasiones, los propios volcanólogos deben facilitar la información de los pronósticos a la prensa y a la población en general, por lo que se resaltó la necesidad de que algunos volcanólogos se especialicen para presentar la información al público como voceros, se concienticen de la importancia de esta labor y desarrollen un uso del lenguaje adecuado para relacionarse con los medios de comunicación. Este último aspecto es de especial relevancia durante épocas de crisis volcánicas debido a la gran demanda de información que se genera.

La relación directa entre volcanólogos y medios de comunicación fue un aspecto que no se debatió en profundidad durante las jornadas, pero se trata de un ámbito donde los profesionales de la volcanología de los diferentes países tienen buenas y malas experiencias, que en futuros foros de debate sería de gran interés compartir. En América Latina, o al menos en la región centroamericana, es poco común que alguno de los integrantes de un medio de comunicación tenga la posibilidad de especializarse en temas técnico-científicos o en periodismo científico. En la medida de lo posible, favorecer la capacitación de los profesionales de la comunicación en este ámbito podría apoyar las tareas de divulgación de los volcanólogos.

En el caso de la relación directa entre volcanólogos y las comunidades sí se mostraron, durante la jornada, diversas experiencias, entre las que cabe resaltar los programas de observadores locales que se desarrollan en diferentes países. En el caso de algunos de los volcanes en Guatemala, tanto el INSIVUMEH como la Coordinadora Nacional para la Reducción de Desastres (CONRED) han desarrollado una estrategia de interacción permanente a nivel local con las comunidades más expuestas a las amenazas. A finales de las décadas de 1990 y principios de 2000, el INSIVUMEH estableció observatorios volcanológicos en algunas de las comunidades cercanas 
a los volcanes Santiaguito, Fuego y Pacaya. Estos observatorios son operados por pobladores de las mismas comunidades, quienes han recibido entrenamiento y son pagados por el INSIVUMEH. Usualmente estos observadores forman parte de la estructura de liderazgo local, y en esta forma se convierten en un vínculo sumamente importante y de gran valor entre las comunidades y el INSIVUMEH.

De forma similar, la CONRED ha establecido una serie más extensa de voluntarios en las comunidades expuestas a la amenaza volcánica, quienes forman parte del sistema comunitario de alerta temprana. Estos voluntarios han recibido entrenamiento, y han sido dotados con equipo de radiotransmisión, para facilitar la comunicación entre ellos y los distintos niveles (local, regional y nacional) de la CONRED. Al igual que con los observatorios del INSIVUMEH, la red de voluntarios se convierte en un vínculo sumamente importante entre las comunidades y la CONRED, además de que hasta cierto punto funcionan como observatorios, al reportar la actividad volcánica que ellos pueden ver. Este esfuerzo se desarrolla dentro de un esquema más general y de gran envergadura, en el cual el CONRED por mandato institucional debe organizar coordinadoras locales en áreas que estén expuestas a la amenaza. Esquemas similares de interacción entre la población y los volcanólogos se desarrollan en Ecuador y en menor medida en El Salvador. De hecho, el nuevo Plan Nacional de Reducción de Riesgo por parte del Ministerio de Medio Ambiente y Recursos Naturales de El Salvador, a través de la DGSNET en El Salvador, ha tomado en cuenta la importancia de vincular el marco institucional del nivel nacional al nivel comunitario, lo cual se traduce en la formación de redes de observadores locales que fungen como fuentes de información in situ de los cambios en el comportamiento de la actividad volcánica y también como enlaces de las instituciones hacía las comunidades.
Este contacto entre las comunidades y las organizaciones estatales involucradas en la gestión del riesgo volcánico ha sido vital durante el manejo de algunas de las crisis eruptivas que han ocurrido recientemente en Guatemala, y ha servido como una estrategia para mejorar la credibilidad y confianza en dichas instituciones dentro de las comunidades. Sin embargo, dadas las limitaciones de recursos, el esquema solamente se ha aplicado a volcanes de conducto abierto, en los cuales la actividad se manifiesta en erupciones continuas o diarias, ya sea efusivas o explosivas de baja intensidad, que en la mayoría de los casos no representan una amenaza a la población, pero que eventualmente cambian a erupciones explosivas más violentas. Existen otras experiencias en el caso de volcanes de conducto abierto que han tenido una actividad más o menos continua, en que la coordinación entre las instituciones y las comunidades no siempre ha resultado en historias exitosas. Durante el taller participativo "Knowledge Sharing and Collaboration in Volcanic Risk Mitigation at Galeras Volcano, Colombia," con representación de instituciones gubernamentales, no gubernamentales, académicas, nacionales, internacionales, y de las comunidades bajo riesgo del Volcán Galeras, discutieron varios retos y experiencias amargas durante el manejo de crisis volcánicas. Tal vez lo más importante en estos foros es empezar la discusión abierta y respetuosa hacia una estrategia y relación más productiva para incluir las opiniones y necesidades de cada actor (García et al., 2009).

En el caso de otros volcanes activos (p. ej. durante el Holoceno), pero que no han tenido actividad reciente y que pueden considerarse como potenciales fuentes de amenaza en un futuro no muy lejano, la atención que se les ha dado ha sido mínima. Es quizás en estos casos donde la interacción entre las autoridades, los volcanólogos y la población será más complicada durante futuras crisis volcánicas y donde las experiencias adquiridas en volcanes con actividad reciente puedan ser un aporte valioso. 


\section{IMPLICACIONES HUMANAS Y SOCIALES DE LAS AMENAZAS VOLCÁNICAS DESDE EL PUNTO DE VISTA DE LA GESTIÓN Y LA PERCEPCIÓN DEL RIESGO}

Se entiende por gestión del riesgo el proceso social complejo que conduce al planeamiento y aplicación de políticas, estrategias, instrumentos y medidas orientadas a impedir en lo posible, reducir, prever y controlar los efectos adversos de fenómenos peligrosos sobre la población, los bienes y servicios y el ambiente (Lavell, 2000). Este proceso social, no es solamente institucional, sino que se construye a partir de las percepciones y conocimientos previos de los individuos y de las comunidades, de sus características socioeconómicas y del grado de uso del territorio que desarrollan en su vida cotidiana, que van a condicionar el grado en que estas querrán cambiar sus actitudes y su accionar frente a fenómenos potencialmente peligrosos.

En este contexto, el trabajo cotidiano de los volcanólogos en el monitoreo y caracterización de la actividad volcánica y su relación con otros fenómenos peligrosos (actividad sísmica, inestabilidad de laderas, p.ej.) forma parte de las estrategias de reducción de riesgo, incrementando el conocimiento sobre las amenazas. Pero es en la tarea de divulgación de las características del territorio, del fenómeno volcánico y de las amenazas asociadas cuando se incide en la reducción de la vulnerabilidad de las personas y las comunidades, en el sentido de que esta tarea forma parte de un proceso educativo más amplio de construcción de ideas en relación con los niveles de riesgo a los que están expuestos, de los niveles de riesgo asumibles y a la ocurrencia de desastres.

Aunque la participación de los volcanólogos en el proceso de reducción de riesgos no es siempre consciente y sistematizada, se ha aprendido en el camino, que es importante considerar la percepción de riesgos y conocimientos previos adquiridos por las personas, que en muchos casos difiere de las ideas que se tienen en los observatorios sobre estos grupos de población. Especialmente, en caso de crisis volcánica, cuando se traslada información sensible, que puede llevar a la evacuación de parte de la población de los lugares donde viven y trabajan, como se discutió anteriormente.

Algunas de las presentaciones y la discusión durante el taller se desarrollaron en torno a las consideraciones del contexto social, económico, político y humano en general, en el cual se dan los problemas de riesgo y crisis volcánicas. Se revisaron y discutieron algunas de las ideas más profundas o "filosóficas" sobre la ontología y epistemología del riesgo. Entre los puntos más salientes se consideraron aquellos aspectos que hacen de la toma de decisiones para la gestión del riesgo volcánico algo tan complejo y difícil, especialmente durante una crisis.

Una decisión relativa a la gestión del riesgo volcánico, por ejemplo declarar un área inhabitable, o recomendar una evacuación durante una crisis volcánica, siempre tiene un impacto, o representa un costo o pérdida para alguien, y en primer lugar para aquellas personas que se ven afectadas directamente por el resultado de esta decisión. Así, en el caso de la declaración de inhabitabilidad de un área, o de la recomendación de evacuación durante una crisis, son los habitantes de estas áreas los que en primera instancia reciben el impacto de la decisión. Una perspectiva ingenua seria considerar que la decisión solo se justifica a posteriori si en efecto un evento letal alcanza el área declarada inhabitable o evacuada, y como resultado de la decisión se lograron salvar vidas. Sin embargo el caso alternativo desde luego también es posible, p. ej.: que se tome la decisión de declarar un área inhabitable o recomendar una evacuación, pero posteriormente no ocurre un evento potencialmente letal.

El elemento fundamental que hace este tipo de decisiones tan complejas y difíciles, es la incertidumbre asociada con los posibles resultados, 
y es aquí donde toda la capacidad y habilidades de los volcanólogos deben enfocarse, cuando se constituyen en asesores para la toma de este tipo de decisiones. Sin embargo, aun con la mayor capacidad y habilidades disponibles hoy en día en el mundo, la incertidumbre no puede eliminarse, y hay buenas razones teóricas para pensar que de hecho esto nunca podría lograrse. El resultado lógico de esto es que las decisiones sobre acciones de gestión de riesgo siempre tendrán que tomarse en un contexto de incertidumbre.

Para paliar el problema, y dadas las limitantes en el conocimiento y la incertidumbre que se han discutido anteriormente, parte de las acciones de gestión del riesgo deben enfocarse en disminuir el impacto que de otra manera deberán absorber las personas directamente expuestas a la amenaza y sus autoridades, incluso si no se da un evento potencialmente letal, pero se ha tomado una decisión de declarar una zona inhabitable o se ha realizado una recomendación de evacuar. Por ejemplo, el proporcionar una alternativa viable para el traslado de una comunidad de una zona declarada inhabitable a otra zona segura, donde la alternativa de vida sea por lo menos tan atractiva para las personas que se trasladan, como lo era en el sitio donde anteriormente residían. $\mathrm{O}$ en el caso de las evacuaciones, proporcionar condiciones de albergue que cubran todas las necesidades de la población, así como de protección para los bienes que se dejan "temporalmente abandonados". Desde luego que en ambos casos también deberá de existir una alternativa económica y productiva para que estas personas puedan desarrollar un medio de vida y óptimamente deberán tomarse en un marco concatenado con gobiernos locales o centrales.

En el mundo real, y dadas las limitaciones de recursos disponibles, en muchos casos es difícil reducir ese impacto de forma significativa, y las alternativas que se ofrecen usualmente no llenan las expectativas de la población afectada. En este punto se vuelve evidente que es también crucial lograr llegar a un consenso o acuerdo, entre las distintas instancias involucradas en el proceso de toma de decisiones (p. ej.: los pobladores del área expuesta, las autoridades políticas a nivel local, regional y nacional, las autoridades de protección civil, y finalmente los volcanólogos) sobre cuál es la decisión correcta a tomar; es decir, idealmente tanto las autoridades, la población y los volcanólogos estarían de acuerdo si determinada situación de riesgo amerita una evacuación o la declaración de un área como inhabitable. Este consenso, acoplado con el mejor esfuerzo de las autoridades y de protección civil para reducir el impacto de la evacuación o el traslado, y el mejor esfuerzo en analizar la amenaza y generar un pronóstico con un mínimo de incertidumbre y un máximo de valor informativo por parte de los volcanólogos, debería de ser la meta del trabajo conjunto de todos estos grupos, en el tema de la toma de decisiones dentro de la gestión del riesgo volcánico.

Ejemplos de casos en Colombia, donde algunas de las comunidades cercanas al volcán Galeras han resistido la decisión gubernamental de declarar las áreas donde ellos residen como inhabitables (García et al., 2009), o el caso de la crisis eruptiva del volcán Tungurahua en 1999 en Ecuador y el subsecuente conflicto entre los pobladores de la ciudad de Baños y las autoridades de defensa civil y los volcanólogos, son algunos de los casos que mejor ilustran la complejidad del problema. En algunas de las participaciones por representantes de estos países, se discutió cuál ha sido el proceso después de que estos conflictos alcanzaron un clímax, y cómo se ha tratado de abordar el problema, para lograr una mejor solución (ver presentaciones de Ecuador y Colombia en Rose \& Palma, 2011).

Así, en el último decenio, ha habido una creciente preocupación, tanto por parte de los observatorios como por los grupos de investigación extranjeros por adaptar los productos de divulgación (mapas, charlas, etc.) a los diferentes grupos de población (por edades; rural-urbana; indígena) con el objetivo de que el proceso de transferencia de información sea lo más eficiente y respetuoso posible con los puntos de vista, cultura y cosmovisión de los diferentes grupos destinatarios.

Estas experiencias permiten resaltar la idea de que el rol de los volcanólogos como divulgadores del fenómeno volcánico y sus peligros requiere de la colaboración con profesionales de las ciencias sociales y la creación de grupos multidisciplinarios. Durante la jornada se puso de 
manifiesto cómo en el ámbito de la volcanología existen todavía reticencias acerca de la validez del trabajo de los profesionales de las ciencias sociales. Sin embargo, la mayor parte de los asistentes expresaron la necesidad de que los volcanólogos en los observatorios se involucren o trasciendan al área de la ciencias sociales ("fuera de sus zonas de confort profesional") para poder entender mejor a la población destinataria, desarrollar acciones en congruencia y contribuir así a validar los estudios sociológicos. La investigación sociológica puede aportar valiosa información sobre los grados de vulnerabilidad de las comunidades, sobre los conocimientos previos adquiridos por estas en relación con el territorio donde viven y orientar de forma más efectiva el diseño de materiales informativos, la ejecución de actividades divulgativas y la implementación de proyectos de reducción y mitigación de riesgos, sean estos impulsados por las propias comunidades, por las instituciones nacionales o por organismos de cooperación.

\section{COMENTARIOS FINALES Y CONCLUSIONES}

\section{Carencia de recursos, cabildeo con institucio- nes en el ámbito regional}

La cantidad de recursos que se han dedicado a la volcanología y a la gestión del riesgo volcánico en América Latina es relativamente limitado y en la mayoría de los casos insuficiente para enfrentar las demandas y necesidades que el área requiere, considerando su alto grado de actividad y la gran cantidad de población que vive cerca de áreas volcánicas activas y potencialmente peligrosas en algunas regiones (Small \& Naumann, 2001; Ewert \& Harpel, 2004). Dentro de esta problemática quizás uno de los temas más críticos, es la presencia de ciudades importantes de la región en áreas que pueden ser impactadas por erupciones volcánicas, incluyendo varias ciudades capitales, como Ciudad de México, Ciudad de Guatemala, San Salvador, Managua, San José y Quito (Chester et al., 2000).

Comparados con sus equivalentes en países desarrollados de Europa, Norteamérica y Asia, los observatorios y las organizaciones que realizan estudios volcanológicos en América Latina cuentan con relativamente pocos recursos técnicos, económicos y humanos, para desarrollar las tareas que por mandato les corresponden.

Esta carencia es en parte debida a la situación general de escasez de recursos de los estados latinoamericanos, y en parte debida a la baja prioridad que se le ha asignado al tema, lo cual sigue siendo uno de los grandes retos de gobiernos e instituciones locales que no pueden enfrentar la alta vulnerabilidad de sus poblaciones (Latin American Economic Outlook, 2012). La baja probabilidad de ocurrencia de eventos volcánicos comparada con la probabilidad de ocurrencia de otros eventos geológicos e hidrometeorológicos que frecuentemente causan daños y víctimas, como las inundaciones y los deslizamientos de tierra o la actividad sísmica, son otra de las causas de que los gobiernos de la región, especialmente en América Central, hayan orientado esfuerzos a fortalecer el recurso humano e instrumental en áreas como la meteorología, la hidrología y el monitoreo sísmico, mas no en el tema volcánico. Sin embargo, las erupciones volcánicas tienen un alto potencial de causar devastación en áreas densamente pobladas.

Entre 1990 y 2011 los desastres causados por otros tipo de amenazas naturales han tenido un mayor impacto, tanto por el número de muertes como por las pérdidas económicas causadas, en los países participantes en el taller PASI (Fig. 2A). Sin embargo, la ocurrencia de un solo evento que afecte un área densamente poblada, puede tener consecuencias devastadoras. Tomando como ejemplo la tragedia del Nevado del Ruiz en Colombia en 1985, al incorporar el impacto de esta erupción a los datos presentados en la figura $2 \mathrm{~A}$ se obtiene un panorama muy distinto (Fig. 2B), alterando radicalmente la proporción del impacto debido a las erupciones volcánicas, al menos en cuanto al número de víctimas mortales.

Es también interesante ver que el impacto económico de otros tipos de desastres sigue siendo dominante con respecto al impacto causado por erupciones volcánicas, incluso si ocurriera un desastre como la tragedia del Nevado del Ruiz. Esto parecería indicar que desde una lógica puramente económica, las erupciones volcánicas quizás no 
A Númerode víctimas mortales

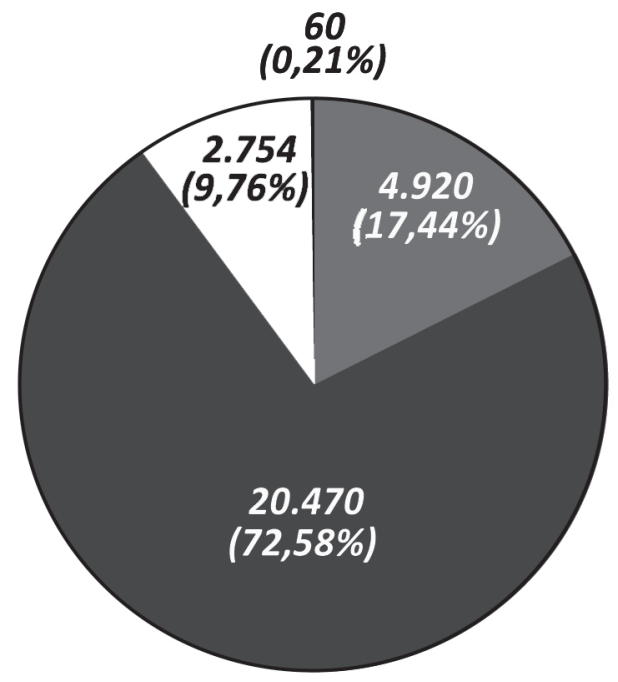

B Número de víctimas mortales

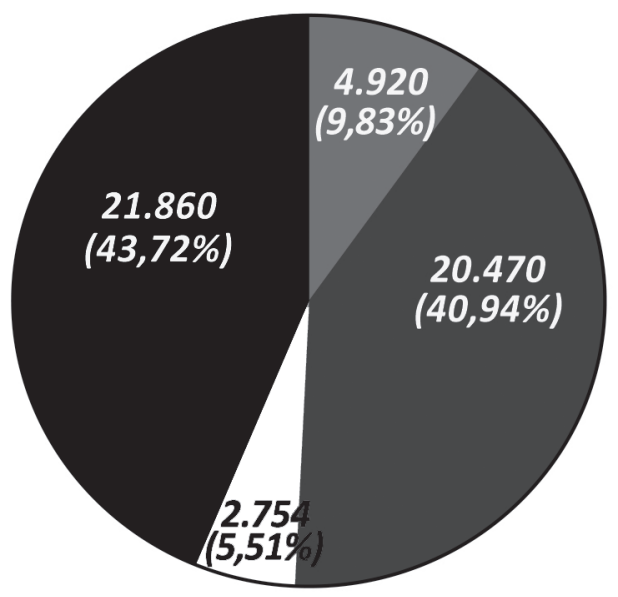

\section{Pérdidas materiales estimadas (USD)}

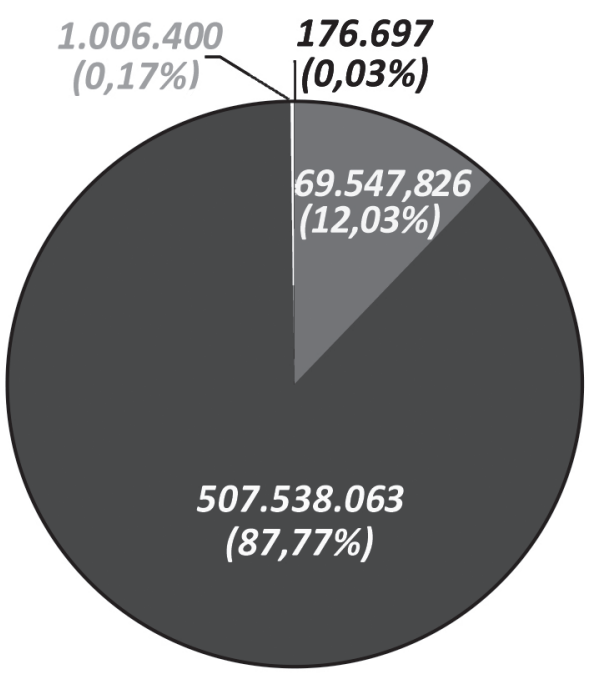

Pérdidas materiales estimadas (USD)

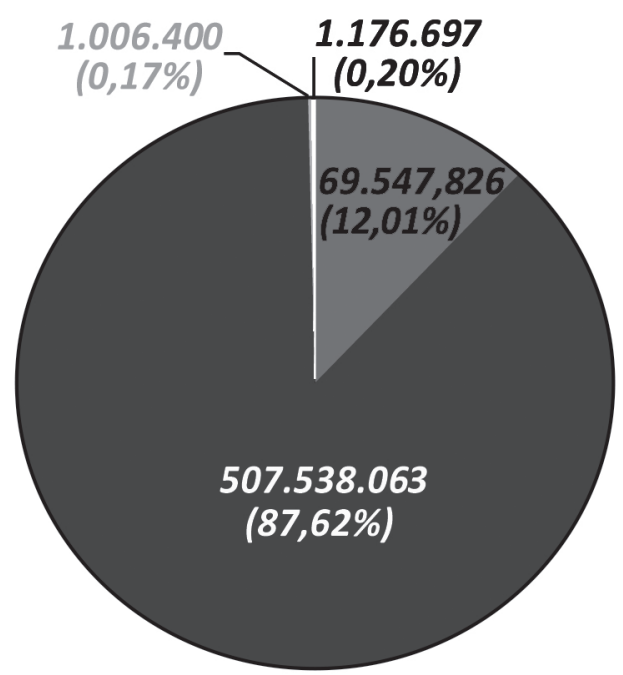

\section{Sismos $\square$ Tormentas e inundaciones $\square$ Deslizamientos $\square$ Volcanes}

Fig. 2: Impacto de los desastres causados por diversas amenazas naturales en los países representados en el taller PASI. A. Impacto agregado de los desastres de 1990 al 2011. B. Impacto de los desastres de 1990 al 2011, agregando los efectos catastróficos provocados por la erupción del Nevado del Ruiz en 1985, en Colombia. Fuente: EM-DAT: The OFDA/CRED International Disaster Database. www.emdat.be - Université Catholique de Louvain - Brussels - Belgium. 
tendrían la misma importancia que desde una lógica "humanitaria". Políticas de desarrollo y financiamiento de los observatorios y las organizaciones que monitorean amenazas naturales, que estén basados solo en el criterio de pérdidas económicas, podrían ignorar el potencial real de los desastres generados por erupciones volcánicas.

Todas estas carencias presentan su aspecto más severo cuando el trabajo está relacionado con la gestión del riesgo, especialmente durante el manejo de las crisis volcánicas, en cuyo caso las consecuencias pueden potencialmente medirse en vidas humanas e infraestructura. Sin embargo, y debido precisamente a esta realidad y a las limitaciones particulares de cada organización en el ámbito de la volcanología que se enfrenta en América Latina, se han desarrollado estrategias altamente innovadoras, basadas en la creatividad y el ingenio que los colegas volcanólogos han demostrado a través de los años.

Estas estrategias involucran el uso más eficiente posible de los escasos recursos, el crear vínculos de cooperación con otras organizaciones que se dediquen a temas afines, ya sea desde el punto de vista de la investigación en el caso de universidades e institutos de investigación, o de la gestión del riesgo, en el caso de grupos de protección civil y ONG. Finalmente, una de las estrategias más interesantes y que merece la pena resaltar, ha sido el involucramiento de la gente en las comunidades cercanas a los volcanes, para realizar tareas de observación visual, como sucede actualmente en Guatemala y Ecuador.

\section{Programas de cooperación técnica y académica}

El carácter eminentemente científico y técnico de muchos aspectos de lo que tradicionalmente ha abarcado la volcanología, ha llevado a que históricamente muchos de los observatorios y de las organizaciones que hacen trabajo en el campo de la volcanología en América Latina hayan tenido una estrecha relación con universidades e institutos de investigación, y en algunos casos, como ocurre en Ecuador, Costa Rica y en Colima (México), hayan surgido del seno de dichas instituciones académicas. Sin embargo, la diversidad del paisaje institucional latinoamericano no permite generalizar esta observación a todos los casos; por ejemplo en algunos países centroamericanos, aunque exista una cooperación esporádica entre las universidades y los observatorios, su desempeño es en su mayor parte independiente y autónomo. Esto debido entre otras razones, y de nuevo, a la carencia de recursos para el fortalecimiento de los equipos de investigación en las universidades centroamericanas, especialmente en las áreas vinculadas a las ciencias de la tierra y en general a una cultura "del corto plazo" vinculada a los tiempos de permanencia de las administraciones, más que a los tiempos de los fenómenos que se estudian. Es el caso, por ejemplo, de El Salvador, donde han existido intentos de coordinación entre el Grupo de Vulcanología de la Universidad Nacional, que realiza sus propias investigaciones sobre emisión de gases volcánicos y la DGSNET; que no lograron consolidarse formalmente. Incrementar la interacción entre ambos tipos de organizaciones podría ser de gran beneficio para todos los involucrados, para complementar las funciones que realizan, para enriquecer la discusión y el análisis de datos, y en consecuencia, los resultados en forma de mejores propuestas de escenarios de amenaza volcánica.

América Latina alberga un gran número y variedad de volcanes (Fig. 1), dispersos un una vasta región geográfica desde México hasta Chile (Corominas \& Martí, 2015), muchos de ellos con frecuente actividad. Esto vuelve a la región un objetivo de interés para investigadores de todo el mundo que desean estudiar diversos tipos de volcanismo, y abre las puertas para la posible colaboración entre estos investigadores y las organizaciones que trabajan el tema volcánico en América Latina. Esta oportunidad ha sido aprovechada 
por muchos años, formando una larga tradición de cooperación entre investigadores de diversas universidades, principalmente de los países más desarrollados, con los observatorios y universidades locales en América Latina. Es el caso del apoyo y fortalecimiento que ha ofrecido por años el Servicio Geológico de los Estados Unidos, a través de su red de observatorios volcanológicos, a diversos países de la región o en el ámbito latinoamericano la colaboración entre el CENAPRED y la Universidad Nacional Autónoma de México.

En muchos casos las relaciones entre los investigadores de organizaciones académicas y los observatorios han sido cimentadas sobre la base de las relaciones personales que individuos en ambas organizaciones ha desarrollado, convirtiéndose esta en una cooperación de facto, que de ninguna manera ha dejado de ser de mucho beneficio. Sin embargo, en muchos casos el establecer relaciones más formales entre instituciones y no solo entre individuos, podría aumentar el potencial de trabajo conjunto y lograr alcanzar de forma más satisfactoria aquellas metas que sean comunes a ambas organizaciones. La falta de un marco institucional de cooperación entre las instituciones académicas y gubernamentales de la región ha impedido la colaboración en algunos casos. Adicional a la creación de un marco institucional de cooperación, es necesario contar con el recurso económico para financiar proyectos de cooperación que requieran no solo de los recursos necesarios para desarrollar el proyecto per se, sino también para financiar los viajes y otros gastos asociados al carácter internacional de este tipo de cooperación.

Aunque las organizaciones académicas y los observatorios en principio comparten muchas metas en el campo de la investigación y el monitoreo volcánico, los objetivos de los primeros en muchos casos tienden a ser menos aplicados o enfocados en los aspectos prácticos (p. ej.: los relacionados con investigación científica "pura") que los objetivos que usualmente persiguen los segundos (p. ej.: los relacionados con la gestión del riesgo). El trabajo cooperativo exitoso entre ambos tipos de organizaciones depende de que las metas comunes se definan de forma clara y con base en ello se establezcan las expectativas del trabajo de cada participante. Esto requiere un diálogo honesto y una negociación sobre el aporte que cada uno hará al trabajo. En algunos casos los observatorios en América Latina apoyan el trabajo de investigadores nacionales o extranjeros a un alto costo de recursos humanos o materiales, sin obtener a cambio un beneficio tangible para los objetivos hacia los cuales debería enfocar su trabajo. Por otro lado, en algunos casos, los investigadores de instituciones académicas que podrían aportar un valioso trabajo de investigación y que sería de interés para los fines del observatorio, reciben poco apoyo de dicho observatorio. Es importante que ambas organizaciones entiendan la perspectiva y los objetivos de la contraparte, para poder plantear objetivos comunes y evitar crear falsas expectativas o frustraciones.

Por otro lado, los observatorios necesitan incrementar el número de profesionales y mejorar su formación (Corominas \& Martí, 2015). Como puede verse en el cuadro 1, el número de observatorios y la cantidad de personal varía ampliamente en el caso de cada país. Por otro lado, el cuadro 2 resume los resultados por país y los compara con el número de volcanes que han tenido actividad en el Holoceno, según el catálogo del Global Volcanism Program (Venzke et al., 2009). La última columna de la Tabla 2 muestra, para cada país, el cociente entre el número de volcanes y el número total de personal que trabaja en los observatorios. Con la excepción de Colombia, Costa Rica y Ecuador, en la mayoría de países latinoamericanos el número de volcanes supera al número de volcanólogos. Tomando a EE. UU. como un parámetro de comparación, la cantidad de volcanólogos trabajando en observatorios supera a la cantidad de volcanes, incluso cuando se toman en cuenta los numerosos volcanes de las regiones relativamente despobladas de Alaska.

Desde luego que este análisis es muy superficial y no toma en cuenta factores más profundos, pero creemos que en todo caso la situación sería aun más crítica para América Latina si se analizaran otros factores, como la inversión económica en equipo de monitoreo, y la participación en el monitoreo de otras organizaciones que no aparecen enlistadas en el catalogo del WOVO, como universidades y centros de investigación. 
Cuadro 1

Personal en los observatorios volcanológicos de América Latina y Estados Unidos hasta 2011

\begin{tabular}{|c|c|c|}
\hline Observatorio / Institución & País & Personal \\
\hline OVDAS & Chile & 7 \\
\hline INGEOMINAS Manizales & Colombia & 16 \\
\hline INGEOMINAS Pasto & Colombia & 17 \\
\hline INGEOMINAS Popayán & Colombia & 12 \\
\hline OSIVAM & Costa Rica & 16 \\
\hline OVSICORI - UNA & Costa Rica & 23 \\
\hline IG - EPN & Ecuador & 33 \\
\hline Alaska Volcano Observatory & EE. UU. & 60 \\
\hline Cascades Volcano Obsevatory & EE. UU. & 51 \\
\hline Hawaii Volcano Observatory & EE. UU. & 24 \\
\hline Yellowstone Volcano Observatory & EE. UU. & 3 \\
\hline UES Instituto de Ciencias de la Tierra & El Salvador & 9 \\
\hline DGSNET & El Salvador & 3 \\
\hline INSIVUMEH & Guatemala & 9 \\
\hline CONRED & Guatemala & 12 \\
\hline Observatorio Vulcanológico / Universidad de Colima & México & 14 \\
\hline CENAPRED & México & 16 \\
\hline INETER & Nicaragua & 5 \\
\hline IGP - Arequipa & Perú & 12 \\
\hline TOTAL & & 342 \\
\hline
\end{tabular}

Fuente: World Organization of Volcanological Observatories. http://www.wovo.org/observatories.html

Para aumentar la capacidad de los observatorios, es necesario que las organizaciones y agencias de monitoreo a nivel nacional en cada uno de los países, destinen mayores recursos a tal fin, sin que estos sean dependientes de la cooperación internacional. Los organismos supranacionales, como el Sistema de Integración Centroamericano (SICA), a través de su Centro para la Prevención de Desastres Naturales en América Central (CEPREDENAC) pueden ser más sensibles a estas necesidades e influenciar a los gobiernos nacionales para que se visualice el volcanismo y las amenazas asociadas como una realidad de los países latinoamericanos y se destine a la reducción del riesgo volcánico, considerada desde una perspectiva de multiamenaza, con recursos similares a los de otros tipos de riesgos.

La inversión en la formación técnica y científica del personal de los observatorios y las organizaciones académicas que los apoyan, llevará en última instancia a la creación de grupos de investigadores profesionales fuertes dentro de estas instituciones. Estos grupos, además de su función docente, tendrían la misión de desarrollar programas de investigación, generar información y conocimiento y de esta forma complementar y fortalecer el trabajo operativo en los observatorios. En este sentido los programas de cooperación técnica, dirigidos en los últimos años principalmente a la implementación y uso de redes de monitoreo y a la capacitación puntual en determinadas técnicas de evaluación de amenazas, deberían reorientarse a programas de cooperación académica para la creación de grupos multidisciplinarios de investigación en las universidades locales. Es posible para este fin aprovechar los vínculos y la relación de trabajo ya establecidos con grupos de investigación norteamericanos y europeos principalmente. 
Cuadro 2

Volcanes holocenos y personal en observatorios hasta 2011

\begin{tabular}{ccccc}
\hline País & $\begin{array}{c}\text { Número de observato- } \\
\text { rios o instituciones }\end{array}$ & Personal & Volcanes holocenos & $\begin{array}{c}\text { Cociente } \\
\text { personal }\end{array}$ \\
\hline Chile & 1 & 7 & 30 & 4,3 \\
Colombia & 3 & 45 & 14 & 0,3 \\
Costa Rica & 2 & 39 & 10 & 0,3 \\
Ecuador & 1 & 33 & 31 & 0,9 \\
EE. UU. & 4 & 138 & $62(+65$ Alaska) & $0,4(0,9$ con Alaska) \\
El Salvador & 2 & 10 & 20 & 2,0 \\
Guatemala & 2 & 21 & 22 & 1,0 \\
México & 2 & 30 & 32 & 1,1 \\
Nicaragua & 1 & 5 & 19 & 3,8 \\
Perú & 1 & 12 & 16 & 1,3 \\
\hline
\end{tabular}

Fuentes Información sobre los observatorios / instituciones: World Organization of Volcanological Observatories. http://www.wovo.org/observatories.html.

Información sobre los volcanes: Global Volcanism Program / Global Volcano List http://www.

volcano.si.edu/world/globallists.cfm

\section{Coordinación regional}

Debido a que los problemas y retos que enfrentan los volcanólogos en América Latina suelen poseer elementos comunes a todos los países y regiones, y debido a la relativa movilidad e intercambio de información entre los volcanólogos latinoamericanos, se han creado en muchos casos, lazos de amistad y cooperación entre observatorios en distintas regiones y países. Congresos, talleres, e incluso crisis volcánicas, han hecho coincidir en un solo lugar a grupos de volcanólogos latinoamericanos, quienes han aprovechado estas oportunidades para iniciar proyectos de cooperación. Y aún a otro nivel, se han dado en algunos casos, convenios formales de cooperación entre observatorios, universidades y otras organizaciones, tanto gubernamentales como no gubernamentales, muchas veces patrocinados por los gobiernos de los países anfitriones o por gobiernos de países amigos.
Sin embargo, los lazos de comunicación e intercambio de información y conocimiento podrían llevarse a niveles mucho mayores, en beneficio de los volcanólogos involucrados. Dada la tecnología y capacidad de comunicación actual, es razonable pensar en la utilización de redes virtuales y foros de discusión por internet sobre temas y problemas que necesiten ser solucionados en casos de observatorios específicos, por ejemplo por medio de la asesoría de colegas con experiencia durante el desarrollo de una crisis volcánica, o en el planteamiento de proyectos de instrumentación.

Este tipo de comunicación necesitaría de una infraestructura mínima, a costos relativamente bajos. Organizar este tipo de sistemas de comunicación e intercambio virtual requeriría de algún ente que promueva la idea y quizás consiga el financiamiento mínimo para establecer la plataforma tecnológica y administrativa. Sin embargo, también debe de considerarse cómo esta idea encaja en la cultura institucional de 
cada organización y país, sobre todo en el caso del manejo de información sensitiva relacionada al riesgo y las crisis volcánicas. En ese sentido existe en muchos casos una extrema cautela y reserva en la forma en la que se intercambia la información con otras instituciones e individuos.

\section{Formación y especialización de los volcanólo- gos como comunicadores}

Aunque la función principal del volcanólogo es "hacer volcanología", se debe reconocer que la información que este genera debe de ser transmitida a un conjunto de usuarios, a quienes va dirigida. Es vital que los volcanólogos conceptualicen el proceso de generación y transmisión de la información, como el proceso de generar un producto (la información) destinado a un usuario, y que son las necesidades de este usuario, las que determinarán la forma y contenido de ese producto. Esto requiere que exista una comunicación abierta y transparente entre el que genera la información (el volcanólogo) y quien la utilizará (el usuario). En ese sentido, el volcanólogo debe aprender a comunicarse con sus potenciales usuarios, primero que nada para conocer las necesidades y discutir qué tipo de información es posible generar. A partir de allí, se debe tratar de adaptar esa información para que su aprovechamiento sea óptimo.

Desde luego, existe un gran número de potenciales usuarios con necesidades muy diversas, por ejemplo el oficial de protección civil que necesita tomar una decisión sobre si recomendará una evacuación o no, durante una crisis, necesita información muy distinta a la que, por ejemplo, necesitan los profesores o maestros de escuelas, sobre la gestión del riesgo volcánico, y que será transmitida a sus alumnos. En ambos casos, los volcanólogos se convierten en proveedores claves de información crucial, pero en los dos casos el contenido y la forma de la información serán muy distintos.
Conocer a los potenciales usuarios de la información y sus necesidades, entablar un diálogo con ellos sobre las mutuas expectativas, y finalmente establecer qué información puede ser de utilidad para ellos, llevará al volcanólogo a ser un comunicador más eficiente.

\section{Interés del desarrollo de estudios sociales y la creación de grupos multidisciplinarios}

El proceso de comunicación de la información sobre los volcanes en general, y sobre el riesgo volcánico y su gestión en particular, pertenece a un conjunto mucho más amplio y complejo de fenómenos sociales, económicos, políticos, culturales, y en una forma general, humanos, en los que el volcanólogo probablemente se verá involucrado debido a los aspectos prácticos de su trabajo. Nuevamente, el caso de la gestión del riesgo volcánico y el manejo de crisis eruptivas es probablemente el caso más importante en esta categoría.

Adquirir un conocimiento básico sobre los aspectos socio-económicos, políticos, culturales, etc., más importantes relacionados con los problemas del riesgo volcánico, puede ser muy importante cuando en algún momento el volcanólogo deba contextualizar la información sobre, por ejemplo, el nivel de amenaza durante una crisis volcánica, en el momento de asesorar a la defensa civil sobre una posible evacuación.

Aunque en parte este tipo de conocimiento nace en muchos casos de forma natural por la interacción que los volcanólogos tienen con las autoridades de defensa civil, las autoridades políticas locales, e incluso la población en las comunidades expuestas a la amenaza, el contar con la perspectiva sistemática, más rigurosa, y por lo tanto técnica y científica de los expertos en las ciencias sociales, humanas, económicas y políticas, puede ser muy importante, aunque sin duda más compleja. 
Sin embargo, aunque algunos volcanólogos decidan sumergirse en las aguas de otras disciplinas relacionadas con las ciencias sociales y afines, en la mayoría de los casos no tendrán la oportunidad o el tiempo de profundizar mucho en esos temas, y pretender que cada profesional adquiera un conocimiento mucho más detallado que el conocimiento básico en estos, simplemente no es una opción realista. Es en este caso donde el papel del trabajo interdisciplinario se vuelve relevante. La colaboración entre volcanólogos que trabajen en los observatorios e instituciones académicas, y los académicos y expertos de áreas de ciencias sociales y afines puede proveer el tipo de asesoría necesaria para resolver los problemas que involucran a ambos campos, como por ejemplo en el caso de la toma de decisiones durante crisis volcánicas.

Este tipo de cooperación ha visto un auge en el mundo académico, y es razonable pensar que se podría replicar fácilmente en la práctica.

\section{AGRADECIMIENTOS}

Este artículo recopila las opiniones vertidas por los asistentes a la jornada de debate sobre el rol de los volcanólogos en la gestión del riesgo volcánico durante el taller "PASI 2011: Volcanes de Conducto Abierto", organizado por la Michigan Technological University y la Universidad de Costa Rica, y financiado por el proyecto $\mathrm{N}^{\circ} 0530109$ de la National Science Foundation de Estados Unidos. Los autores agradecen la participación en esta jornada de Martha Navarro y Angélica Muñoz (INETER, Nicaragua), Milton Ordóñez (INGEOMINAS, Colombia), Jorge Bustillos y Silvia Vallejo (ING, Ecuador), Nick Varley (Universidad de Colima, México), Hugo Delgado Granados y Ramón Espinasa (Universidad Nacional Autónoma de México), Francisco Montalvo (DGSNET, El Salvador); Guillermo E. Alvarado y Mauricio M. Mora (Universidad de Costa Rica); María Martínez (Universidad Nacional, Costa Rica); Natalia Ruiz (Universidad de Panamá); Riky Centeno, Edu Luis Taipe y Pablo Masías (INGEMMET, Perú); Álvaro Amigo y Gabriel
Orozco (SERNAGEOMIN, Chile); Lizzette Rodríguez (Universidad de Puerto Rico); William Rose y Anieri Morales (Michigan Technological University) y José Luís Palma (VHUB, University at Buffalo). Agradecimientos también para el Ing. Enrique Guevara, a Gerardo Soto y Carlos Navarro por la revisión detallada del manuscrito, que permitió mejorarlo sustancialmente.

\section{REFERENCIAS BIBLIOGRÁFICAS}

BOWMAN, L. \& WHITE, P., 2012: 'Community' perceptions of a disaster risk reduction intervention at Santa Ana (Ilamatepec) Volcano, El Salvador.- Environ. Hazards Spec. Issue: Disaster Risk Reduction for Natural Hazards: Putting Research into Practice: 1-17.

CHESTER, D. K., DEGG, M., DUNCAN, A. M. \& GUEST, J. E, 2000: The increasing exposure of cities to the effects of volcanic eruptions: a global survey. Global environmental change Part B.- Environ. Hazards, 2(3): 89-103.

COROMINAS, O. \& MARTÍ, J., 2015: Estudio comparativo de los planes de actuacion frente el riesgo volcánico (Costa Rica, El Salvador, Ecuador, España, México, Nicaragua y Chile).- Rev. Geol. Amér. Central, 52: 33-56.

DELGADO-GRANADOS ET AL., 2015: - Revista Geológica de América Central, 52: 57-66.

EWERT, J. W. \& HARPEL, C. J., 2004: In Harm's way: Population and volcanic risk.- Geotimes, 4: 14-17.

GARCÍA, C., BOWMAN, L., WILMSHURST, J., RUIN, I., ESCOBAR-WOLF, R., BAJO, J. \& CÓRDOBA, G., 2009: Experiences derived from the participative workshop: Knowledge sharing and collaboration in volcanic risk mitigation at Galeras volcano, Colombia.- Firsts World 
Conference Volcanoes, Landscapes and Cultures Conference. Nov. $4^{\text {th }}-6^{\text {th }}$. Catania, Italy: 55-56.

\section{LATIN AMERICAN ECONOMIC OUTLOOK} (2012): "Decentralisation." Latin American Economic Outlook 2012: Transforming the state for development.- http://www.oecd. org/dev/americas/48965859.pdf [Consulta: 20 de enero de 2012].

LAVELL, A., 2000: Desastres y Desarrollo: Hacia un Entendimiento de las Formas de Construcción Social de un Desastre: El Caso de Mitch en Centroamérica. - Centro Regional sobre Desastres, América Latina y el Caribe (CRID).- http://http://www. desenredando.org/public/articulos/2000/ dyd/DyD2000_mar-1-2002.pdf [Consulta: 1 de agosto de 2011$]$.

NEWHALL, C., ARAMAKI, S., BARBERI, F., BLONG, R., CALVACHE, M., CHEMINÉE, J., PUNONGBAYAN, R.,
SIEBE, C., SIMKIN, T., SPARKS, S. \&, TJETJEP, W., 1999: Professional conduct of scientists during volcanic crises.- Bull. Volcanol., 60(5): 323-334.

ROSE, W. I. \& PALMA, J. L., 2011: PASI, Volcanic Hazards and Remote Sensing in Pacific Latin America Workshop, Costa Rica.- https://vhub.org/resources/303 [Consulta: 2 de agosto de 2011].

SMALL, C. \& NAUMANN, T., 2001: The global distribution of human population and recent volcanism.- Environ. Hazards, 3: 93-109.

VENZKE, E., WUNDERMAN, R.W., MCCLELLAND, L., SIMKIN, T., LUHR, J.F., SIEBERT, L., MAYBERRY, G. \& SENNERT, S. (eds), 2009: Global Volcanism, 1968 to the Present.Smithsonian Institution, Global Volcanism Program Digital Information Series, GVP4.- http://www.volcano.si.edu/ [Consulta: 2 de agosto de 2011]. 